\title{
An Exploration of Ethnic and National Identity Development in Palestinian Youth in the West Bank
}

\author{
Haneen A. Haddad, PsyD \\ Massachusetts School of Professional Psychology
}

Background: Childhood and adolescence are times of growth and development, regardless of a child's ethnicity, heritage, or circumstances. Cognitive, emotional, and social developments facilitate the development of a psychosocial identity. Until recently, models of identity development have not taken into account the impact that culture can have on a child. Psychologists must begin to consider the impact that culture, environment, and family have on youths' development of identity. This is especially true for the study of ethnic identity development. Furthermore, studies on ethnic identity should focus on particular ethnic groups, issues of social class, and issues related to the social environment (whether one's region is peaceful or war torn), as developmental trajectories and conceptual models may vary depending on all these factors. The goal of this research was to address the gaps in the literature on ethnic identity development in Palestinian youth.

Methods: Sixteen children between the ages of 10 and 16 residing in either a refugee camp or a village adjacent to the Israeli separation wall were asked to complete questionnaires exploring their experiences as a child growing up in the West Bank. All children provided written narratives, as well as responding to 10 multiple-choice questions and providing demographic information. Comparisons were made across age groups (i.e., 10-12 or 13-16), genders (i.e., male or female) and locations (i.e., refugee camp or village).

Results: Fifteen participants identified themselves on the basis of a collective identity, with equal numbers of preadolescents and adolescents identifying in this way. Quantitative comparisons between groups demonstrated that chil- 
dren who resided in a refugee camp reported feeling more strongly connected to their ethnic identity than did children from the village $(p=0.003)$. Participants from the refugee camp were more likely to define themselves only on the basis of their national identity, while villagers more frequently described themselves on the basis of multiple collective identities (i.e., national, ethnic, and religious). Boys tended to have more to say than girls, although the two most prolific writers were girls. In particular, boys more frequently discussed the importance of resistance to oppression than girls. Unsurprisingly, adolescents tended to have a more complex and nuanced sense of collective identities than preadolescents.

Conclusions: Future research should further address the development of collective identity in this population, particularly through longitudinal studies incorporating the use of interviews with more diverse participants, including Palestinian Israelis, Christians, and Druze. Psychologists working with this population must be aware of, and foster, positive developmental trajectories, as today's Palestinian will develop into the leaders and policymakers of the future. 